cause of death. The pathogenesis of SSc-related cardiomyopathy is poorly understood. New therapies as well as platforms for testing are needed. There, the authors characterise different murine models of SSc and identify the best animal model for SSc-related cardiomyopathy.

Methods Six patients with definite SSc were enrolled in the project. Three systemic disease models of scleroderma were chosen: Fra-2 transgenic mice (Fra-2), sclerodermatous chronic Graft versus Host disease (cGvHD) and tight skin 1 mutation (Tsk-1) models. Heart sections were stained for $\alpha$-SMA, CD31, active caspase3, picrosirius, haematoxylin/eosin.

Result Significant accumulation of collagen was observed in hearts of SSc patients. Fibrotic area was increased in $8.8 \pm 2.3$ folds compared to controls $(\mathrm{p}<0.001)$. Same pattern was found in Fra-2 mice with $8.9 \pm 2.8$ fold increase $(p=0.0002)$. No significant fibrosis observed in cGvHD and Tsk-1 mice.

SSc samples presented with increased numbers of myofibroblasts $2.5 \pm 0.6$ vs. $0.5 \pm 0.8(\mathrm{p}=0.01)$ per high power field in controls. Same pattern found in all models of SSc, though highest mimicry observed in Fra- 2 model $(3.0 \pm 1.1$ vs $0.5 \pm 0.6$ $\mathrm{p}=0.0005)$. Slight increases in myofibroblast numbers were also obtained in cGvHD and Tsk-1 models.

CD31 staining revealed significant loss of capillaries in SSc hearts $(2.16 \pm 0.17$ fold change vs controls $p<0.0001)$. Similar capillary loss was observed in Fra- 2 tg and in cGvHD models $(2.0 \pm 0.2$ and $2.1 \pm 0.3$ respectively, $\mathrm{p}<0.0001)$. No changes observed in Tsk-1 mice.

Endothelial apoptosis was increased in Fra-2 tg mice versus controls $(2.5 \pm 0.9$ vs $0.17 \pm 0.43 p=0.0001)$. Apoptosis was observed in cGvHD mice though it was also elevated in controls (2.6 \pm 0.7 vs $1.2 \pm 0.5 \mathrm{p}=0.003)$. No apoptosis detected in Tsk-1 mice. Endothelial apoptosis was also elevated in SSc hearts with $1.2 \pm 0.4$ versus $0.2 \pm 0.4$ in controls ( $p=0.0018$ ). Detected higher numbers of perivascular leucocytes in SSc patients $(5.8 \pm 1.8$ fold change, $p=0.0001)$, strengthened the hypothesis of vascular damage.

Conclusion The typical features of cardiac disease in SScloss of capillaries due to endothelial apoptosis and fibrosis- are closely mimicked by Fra-2 tg mice. In contrast, the changes in $\mathrm{cGvHD}$ and Tsk-1 mice were less representative. Thus, Fra-2 tg mice are a promising preclinical model to study the mechanisms and therapeutic approaches of scleroderma heart involvement.

\title{
10 HEART INVOLVEMENT IN PATIENTS WITH SYSTEMIC SCLEROSIS IS MIMICKED BY FRA-2 TRANSGENIC MICE
}

PVenalis, 0 Distler, ${ }^{2}$ I E Lundberg, ${ }_{1}^{1} \mathrm{G}$ Schett, ${ }^{3} \mathrm{~J} \mathrm{H}$ Distler ${ }^{3}{ }^{1}$ Department of Medicine, Rheumatology Unit, Karolinska University Hospital Solna, Karolinska Institute, Stockholm, Sweden; ${ }^{2}$ Center of Experimental Rheumatology, University Hospital Zurich, Zurich, Switzerland; ${ }^{3}$ Department of Internal Medicine III and Institute for Clinical Immunology, University of Erlangen-Nuremberg, Erlangen, Germany

10.1136/annrheumdis-2011-201235.10

Intoduction Systemic sclerosis (SSc) is a systemic autoimmune disease with vascular and fibrotic components. SScrelated cardiomyopathy is increasingly recognised as a major 